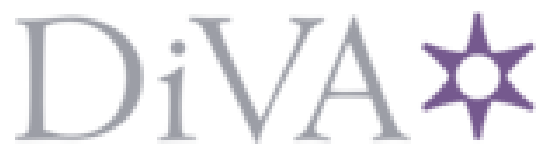

http://www.diva-portal.org

This is the published version of a paper presented at 3oth European Safety and Reliability Conference and 15th Probabilistic Safety Assessment and Management Conference (ESREL2O2O PSAM15).

Citation for the original published paper:

Wang, R., Leander, J., Karoumi, R. (2020)

Risk Analysis for Decision Support --- A Case Study on Fatigue Assessment of a Steel Bridge

In:

N.B. When citing this work, cite the original published paper.

Permanent link to this version:

http://urn.kb.se/resolve?urn=urn:nbn:se:kth:diva-277021 


\title{
Risk analysis for decision support — a case study on fatigue assessment of a steel bridge
}

\author{
Ruoqi Wang \\ Division of Structural Engineering and Bridges, KTH Royal Institute of Technology, Sweden. \\ E-mail: ruoqi@kth.se \\ John Leander \\ Division of Structural Engineering and Bridges, KTH Royal Institute of Technology, Sweden. \\ E-mail: john.leander@byv.kth.se \\ Raid Karoumi \\ Division of Structural Engineering and Bridges, KTH Royal Institute of Technology, Sweden. \\ E-mail: raid.karoumi@byv.kth.se
}

Fatigue is among the most critical forms of deterioration damage that occurs to steel bridges. It causes a decline of the safety level of bridges over time. Much research has been accomplished on a reliability level, which focuses on evaluating the service life of the selected detail or the whole structure. However, the decisions aiming to keep the existing bridges in service should be further considered, i.e. whether interventions are needed, what kind of interventions could be applied, which intervention is the optimal choice to be both sustainable and economical within a budget restraint considered. Therefore a risk-based decision making framework is necessary for bridges service life extension. In this paper, a risk-based decision support framework is formulated aiming for rational decisions, which is presented as a decision tree. It presents possible intervention actions, evaluates the corresponding probability of failure and integrates them with possible consequences. A case study of the Rautasjokk Bridge in Sweden is incorporated to demonstrate the framework. A separation of the load bearing structure in subsystems is suggested for risk analysis and decision optimization. The expected result and how to prioritize the calculated utilities are discussed. And the results are compared with the reliability analysis to check if they are consistent.

Keywords: Risk analysis, decision-making, decision tree, fatigue assessment, subsystem, steel bridge, service life extension.

\section{Introduction}

Fatigue is among the most critical forms of deterioration damage that occurs to steel bridges. It's usually causing a decline of the safety level over time due to repeated or varying loading. Therefore, the performance of steel bridges, which may be seriously affected due to fatigue, should be assessed and predicted.

Much work has been accomplished in the reliability level (Kwon and Frangopol (2010); Leander and Al-Emrani (2016); Schneider et al. (2017)). When the selected structure falls below the target reliability, a predefined acceptable performance level, it's regarded as failure. Then corrective maintenance, performed after a real failure, should be implemented.

Compared to corrective maintenance, preventive maintenance is more preferred to effectively avoid failure and reduce the amount of possible cost. It's essential to decide in advance what types of interventions could be applied as preventive maintenance and which intervention is the optimal choice to be both sustainable and economical. For this reason, risk analysis should be applied for the fatigue assessment of steel bridges and supporting decision-making. A decision tree is established to show the evaluation graphically. Decision tree is a decision support tool that uses a tree-like graph or model of decisions and their possible consequences, including chance event outcomes, resource costs, and utility (Haimes (2005)).

The Rautasjokk Bridge, a railway steel bridge in the northern Sweden, was selected as a case study. A series subsystem extracted from the load bearing structure was evaluated by risk analysis and demonstrated by the decision tree. The possible scenarios were evaluated in probability and consequences respectively. The decisions based on reliability analysis and risk analysis were compared if they're consistent with each other. 


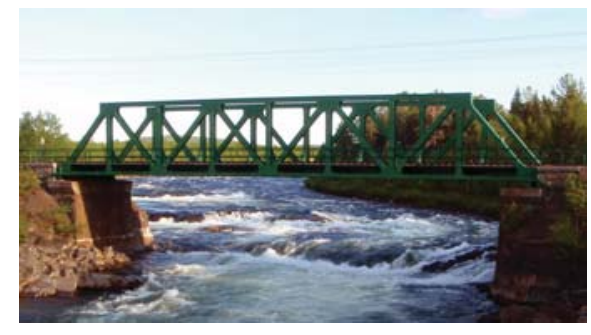

Fig. 1. A photo of the Rautasjokk Bridge

\section{Fatigue assessment}

\subsection{Probabilistic model}

The initiation and propagation of a fatigue crack is a strongly non-linear phenomenon considering different levels of uncertainty. To avoid unduly conservatism by a deterministic approach, a probabilistic model was established. It is based on linear elastic fracture mechanics (LEFM) and Paris law for crack growth.

Paris (1961) proposed a crack-growth model, which describes the relationship between cyclic crack growth and the stress intensity factor range:

$$
\frac{d a}{d N_{\mathrm{r}}}=A K_{\mathrm{r}}^{m}
$$

where $a$ is the crack size, $N_{\mathrm{r}}$ is the number of stress cycles, $A$ and $m$ are fatigue growth parameters. The variable $K_{\mathrm{r}}$ is the stress intensity factor range, depending on the stress range $S$, the crack size and the geometry of the considered detail. $K_{\mathrm{r}}$ is expressed as:

$$
K_{\mathrm{r}}(a)=S \sqrt{\pi a} Y(a) M_{\mathrm{k}}(a)
$$

where $Y(a)$ is a geometry correction factor considering the geometry of the unwelded component, and $M_{\mathrm{k}}(a)$ is a stress magnification factor due to the weld geometry (Hobbacher (1992)).

For the analyses presented in this paper, a bi-linear crack growth rate relation have been adopted as suggested in BS 7910:2013 (BSI (2013)). It is expressed as:

$$
\frac{d a}{d N_{\mathrm{r}}}=\left\{\begin{array}{cc}
0 & K_{\mathrm{r}}<K_{\mathrm{th}} \\
A_{\mathrm{a}} K_{\mathrm{r}}^{m_{\mathrm{a}}} & K_{\mathrm{th}} \leq K_{\mathrm{r}}<K_{\mathrm{ab}} \\
A_{\mathrm{b}} K_{\mathrm{r}}^{m_{\mathrm{b}}} & K_{\mathrm{r}} \geq K_{\mathrm{ab}}
\end{array}\right.
$$

where $K_{\text {th }}$ is the crack growth threshold below which $K_{\mathrm{r}}$ causes no crack growth. The transition point $K_{\text {ab }}$ is calculated as:

$$
K_{\mathrm{ab}}=\left(\frac{A_{\mathrm{a}}}{A_{\mathrm{b}}}\right)^{1 /\left(m_{\mathrm{b}}-m_{\mathrm{a}}\right)}
$$

The bi-linear crack growth law in Eq.(3) is plotted in Figure 2 with mean and 95\% confidence interval. The characteristic values of each stochastic variable considered can be found in Table 1.

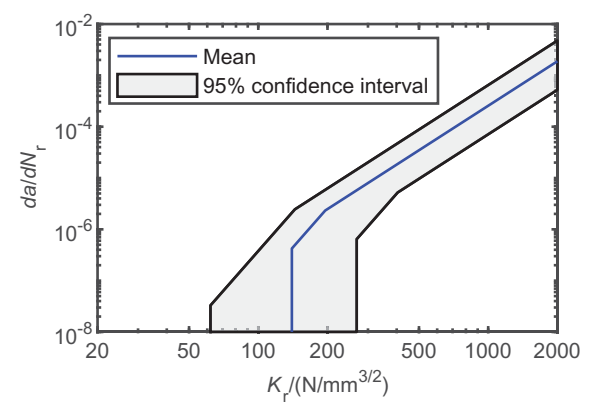

Fig. 2. Bi-linear crack growth law

Based on the crack growth law, the number of cycles to reach a critical crack depth is calculated as:

$$
N_{\mathrm{c}}(\mathbf{x})=\int_{a_{0}}^{a_{\mathrm{cr}}}\left(\frac{d a}{d N_{\mathrm{r}}}\right)^{-1} d a
$$

where $a_{0}$ is the initial crack size and $a_{\mathrm{cr}}$ is the critical crack size. The vector $\mathrm{x}$ contains the stochastic variables considered in the probabilistic model.

\subsection{Reliability assessment}

A general limit state function (LSF) considering fatigue for one single detail is formulated on the number of cycles as:

$$
g(\mathbf{x}, t)=N_{\mathrm{c}}(\mathbf{x})-N_{\mathrm{s}}(t)
$$

where $N_{\mathrm{c}}(\mathbf{x})$ is described by Eq. $(5)$ and $N_{\mathrm{s}}(t)$ is the number of cycles at the studied point in time.

A series system is also regarded as a nonredundant system, which means single detail's failure will lead to the failure of the whole system. The LSF for the series system is modified as:

$$
g(\mathbf{x}, t)=\min \left\{N_{\mathrm{c} i}(\mathbf{x})-N_{\mathrm{s}}(t)\right\}
$$

where $i$ refers to the number of details in this system.

Reliability analysis is concerned with the evaluation of the probability of failure, defined as:

$$
P_{\mathrm{f}}=P(g(\mathbf{x}) \leq 0)=\int_{g(\mathbf{x}) \leq 0} f_{\mathbf{x}}(\mathbf{x}) d \mathbf{x}
$$

where $f_{\mathbf{x}}(\mathbf{x})$ is the joint probability density function (PDF) of all stochastic variables contained in $\mathbf{x}$, and $g(\mathbf{x}) \leq 0$ represents the region of failure in $\mathbf{X}$-space.

If the theoretical assessment is based on a measured indication of damage, the prior estimation of the reliability can be updated considering results from inspection. The detection event can be established as:

$$
H_{\mathrm{D}}(\mathbf{x})=a\left(\mathbf{x}, N_{\mathrm{ri}}\right)-a_{\mathrm{d}}
$$


Table 1. Stochastic variables. $\mathrm{LN} \sim$ Lognormal distribution, DET $\sim$ Deterministic value. Crack growth $[\mathrm{mm} /$ cycle $]$, stress intensity - $[M P a \sqrt{\mathrm{mm}}]$.

\begin{tabular}{|c|c|c|c|c|c|}
\hline \multicolumn{2}{|c|}{ Variables } & Distri. & Mean & $\mathrm{CoV}$ & Correlation \\
\hline$C_{\mathrm{S}}$ & Model & LN & 1 & 0.03 & \multirow{2}{*}{0.2} \\
\hline$C_{\mathrm{SIF}}$ & uncertainties & LN & 1 & 0.07 & \\
\hline$\Delta \sigma$ & Stress range & DET & - & - & - \\
\hline$A_{\mathrm{a}}$ & \multirow{5}{*}{$\begin{array}{c}\text { Material } \\
\text { parameters }\end{array}$} & $\mathrm{LN}$ & $4.80 \cdot 10^{-18}$ & 1.70 & \multirow{3}{*}{1} \\
\hline$A_{\mathrm{b}}$ & & $\mathrm{LN}$ & $5.86 \cdot 10^{-13}$ & 0.60 & \\
\hline$K_{\text {th }}$ & & LN & 140 & 0.40 & \\
\hline$m_{\mathrm{a}}$ & & DET & 5.10 & - & - \\
\hline$m_{\mathrm{b}}$ & & DET & 2.88 & - & - \\
\hline$a_{0}$ & $\begin{array}{c}\text { Initial } \\
\text { crack size }\end{array}$ & $\mathrm{LN}$ & 0.15 & 0.66 & 0 \\
\hline$a_{\mathrm{cr}}$ & $\begin{array}{c}\text { Critical } \\
\text { crack size }\end{array}$ & DET & $\mathrm{w} / 2$ & - & - \\
\hline
\end{tabular}

where $a\left(\mathbf{x}, N_{\text {ri }}\right)$ is the estimated crack size at cycle $N_{\mathrm{r} i}$, and $a_{\mathrm{d}}$ is the lower bound limit of detectable crack size descried by a probability of detection (PoD) curve. Then the estimated probability of failure can be updated assuming no crack is detected $\left(H_{\mathrm{D}} \leq 0\right)$ as:

$$
P_{\mathrm{f}}^{\text {up }}=P\left[g(\mathbf{x}) \leq 0 \mid H_{\mathrm{D}}(\mathbf{x}) \leq 0\right]
$$

\subsection{A theoretical model of the decision tree}

To avoid failure and limit the potential cost, decision makers should consider that there are a number of possible interventions based on the current condition of the selected structure and be able to determine which intervention is suitable in a specific case. In order to help them make an optimal decision, the risk analysis was introduced, which evaluates both the probabilities and possible consequences. A decision tree model, which has been specified for the fatigue assessment, was established with several possible scenarios as shown in Figure 3.

The decision tree is based on a reference time frame. Based on the status of the current time point, the so-called initial condition, different scenarios can be followed and an optimal decision will be made for a future time point.

- $a_{0}$ : do nothing.

The prior reliability assessment of the selected structure should be performed, which estimates the probability of failure at the studied time point. This scenario is justified if the initial condition determines that the structure does not, within a reference time frame, require any more attention and that no immediate action is required.

For instance, if the prior reliability assessment estimates that the reliability of the selected structure still has some margin above the target value at the studied time point, no extra intervention is needed. However, it would be difficult to apply this criteria for a system assessment, since there is no defined target system reliability.

- $a_{1}$ : direct repair.

Here refers to implement strengthening to the selected structure without any inspection performed. This scenario could be an option if the prior assessment shows an exhausted fatigue life, a direct repair could efficiently increase the fatigue resistance.

For example, for welded structures increasing the cross-sectional dimensions or improving the weld geometry will efficiently reduce the stress range at the critical details, which will further improve the fatigue resistance.

- $a_{2}$ : inspection.

This scenario is applied if the prior assessment indicates an exhausted fatigue life, but more information about the damage state is required before making any decisions. An inspection brings new knowledge and it corresponds to the posterior reliability assessment.

There are various methods of performing an inspection, and they achieve different accuracy level. In the following case study, visual inspection is selected due to its simplicity and lowcost in practice.

\section{Case study}

\subsection{Description of the system}

The Rautasjokk Bridge is located along the iron ore railway line in Northern Sweden, between Kiruna and Abisko. A photo of the bridge is shown in Figure 1. The load carrying structure consists of two simply supported parallel steel trusses, with a span length of 33 meters. The single track is carried by stringer beams, which in turn spans between crossbeams. The Rautasjokk Bridge is selected as a case study because it's representative for many similar railway bridges in Sweden and globally. And it also showed strong indications of an exhausted fatigue life due to 


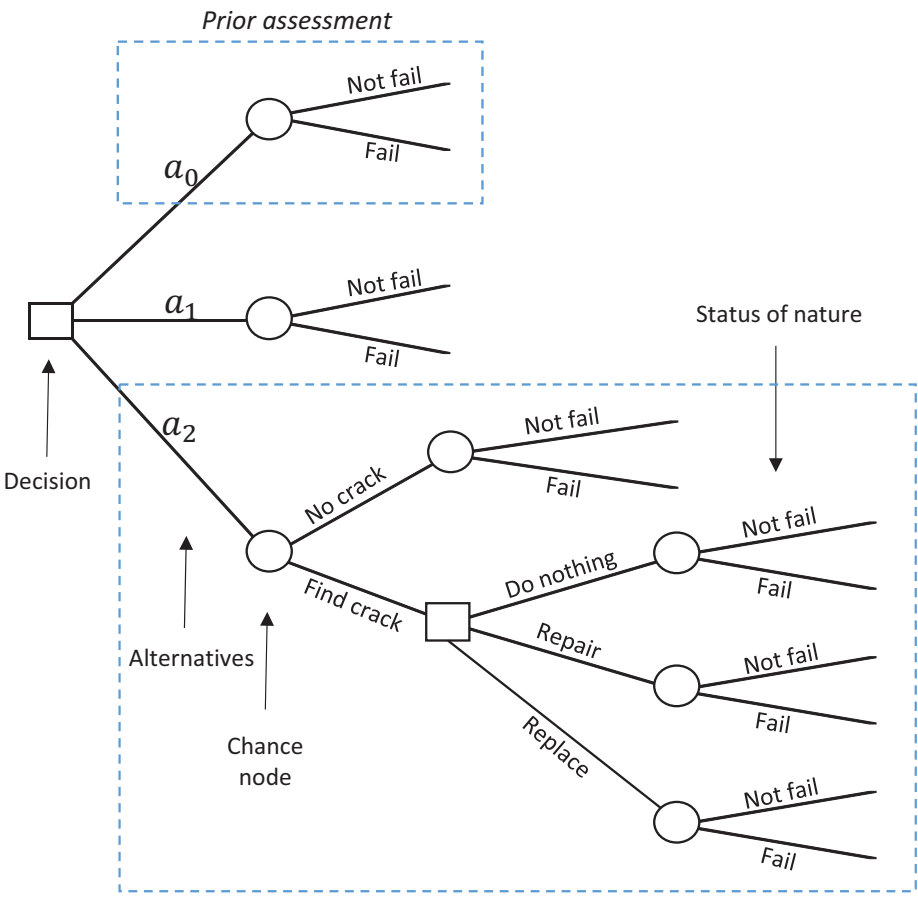

Posterior assessment

Fig. 3. A decision tree model

high load levels from iron ore trains (Häggström (2015)).

The monitoring campaign of the Rautasjokk Bridge consists of six uni-axial strain gauges. Their positions are shown in Figure 4. A codebased assessment of the individual details has been performed by Häggström (2015) showing that gauges 101 and 102 located close to the gusset plate on the stringer beam have the most critical reliability status. Figure 5 shows the dimensions of the gusset plate, which is prone to failure. A crack is assumed to initiate and propagate into the flange at the end of a welded on in-plane gusset plate. For this detail, $Y(a)$ is defined by Newman Jr and Raju (1981) and $M_{\mathrm{k}}(a)$ is given by Leander et al. (2013).
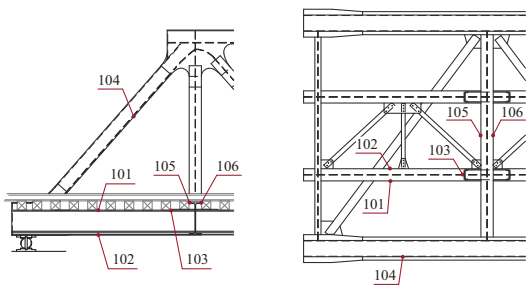

Fig. 4. The location of strain gauges

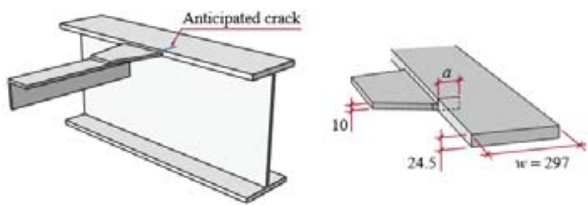

Fig. 5. Dimensions and crack configurations of the gusset plate

There are eight similar details on the bridge, as shown in Figure 6. They are modelled as a series system, which implies that the failure of any detail will lead to a system failure. According to the regulation of Swedish Traffic Administration (Trafikverket), a detected fatigue crack in one of the main load carrying members will be classified as Condition Class 3, which implies immediate action is required. The bridge will not be allowed to continue its service. Besides, the safety of passengers is the prioritized consideration and can't be compromised. Fixing a detected defect at an early stage will guarantee the safety and avoid more severe consequences. Therefore, a noredundancy system model is chosen.

The system reliability analysis was performed considering the probabilistic model in Section 2.1. 


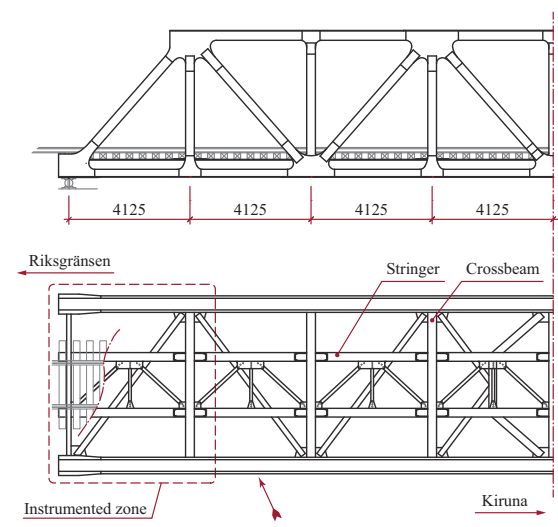

Fig. 6. Series system in the Rautasjokk Bridge

It's assumed that all details are equicorrelated with the correlation coefficient $\rho$. The value is dependent on the correlations of underlying random variables contributing to the failure (Ditlevsen and Madsen (1996)). The stochastic variables and their spatial correlation between different locations are listed in Table $1 . C_{\mathrm{S}}$ and $C_{\mathrm{SIF}}$ with the expected values of unity represent model uncertainties for the stress range and the stress intensity factor respectively. The other variables are selected as suggested in JCSS (2011).

\subsection{Possible scenarios}

Based on the theoretical model in Figure 3, the decision tree was established for the selected subsystem. One year is used as the reference time frame, which means an optimal decision is needed for year 2020 based on the initial condition of year 2019.

\subsubsection{Prior probabilities}

With time passes, the stress cycles accumulate and the system reliability is decreasing. By assuming the traffic conditions in the history being the same as the monitoring result, the accumulated cycle numbers are estimated approximately as $N_{2019}=$ $12.67 \times 10^{5}$ and $N_{2020}=12.89 \times 10^{5}$. A prior reliability assessment was performed. Here the results are shown in Figure 7.

After one year traffic, the reliability of the subsystem is reduced from 2.40 to 2.37 , which corresponds to an increase in the probability of failure from $8.2 \times 10^{-3}$ to $8.9 \times 10^{-3}$.

\subsubsection{Posterior probabilities}

The accuracy of visual inspection has been researched by Campbell et al. (2019). They had invited a diverse group of 30 inspectors to complete a full day, hands-on inspection of 147 steel bridge specimens to establish a quantitative measure of

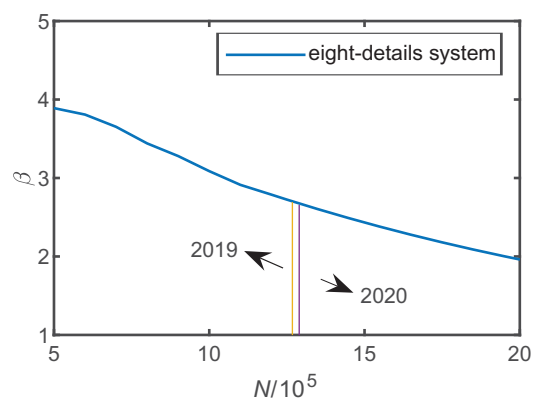

Fig. 7. The prior reliability assessment of the subsystem

visual inspection capability for fatigue cracks in steel bridges. The average detection rate was $65 \%$.

Therefore a 0.65 has been applied in Table 2, representing as for 'a true state of failure, the inspection will indicate a damage' with a likelihood $65 \%, P\left(d_{1} \mid \theta_{1}\right)=0.65$, typically called a true positive.

Table 2. Detection likelihoods for the case study

\begin{tabular}{|c|c|c|}
\hline \multirow{2}{*}{ Inspection result $d$} & \multicolumn{2}{|c}{ Detail state $\theta$} \\
\cline { 2 - 3 } & No failure $\theta_{0}$ & Failure $\theta_{1}$ \\
\hline Indicate no damage $d_{0}$ & 0.65 & 0.35 \\
\hline Indicate damage $d_{1}$ & 0.35 & 0.65 \\
\hline
\end{tabular}

The posterior assessment with inspection may update the probability of failure, as shown in Figure 8 . As the grey boxes indicate, the extra information from the inspection could decrease the probability of failure to a minimum $P\left(\theta_{1} \mid d_{0}\right)=$ $4.8 \times 10^{-3}$, which corresponds to a reliability index of $\beta=2.59$. It represents the event that 'under the condition that no crack is detected, the selected structure fails' with a likelihood of $4.8 \times 10^{-3}$. Correspondingly, the probability of 'under the condition that a fatigue crack is detected, the selected structure fails' increases to $P\left(\theta_{1} \mid d_{1}\right)=1.64 \times 10^{-2}$, which corresponds to a reliability index of $\beta=2.13$.

On the reliability level, the optimal decision is the option which maximizes the reliability of the selected structure. Therefore if a crack is detected $\left(P\left(d_{1}\right)=0.3527\right)$, a replacement will be implemented to give the highest reliability. If no crack is detected $\left(P\left(d_{0}\right)=0.6473\right)$, a direct repair will be implemented. However, the decision based only on the value of reliability could lead to unnecessary construction and may induce a waste of time, money and resources. The consequences of different interventions should be evaluated in the assessment as well. 


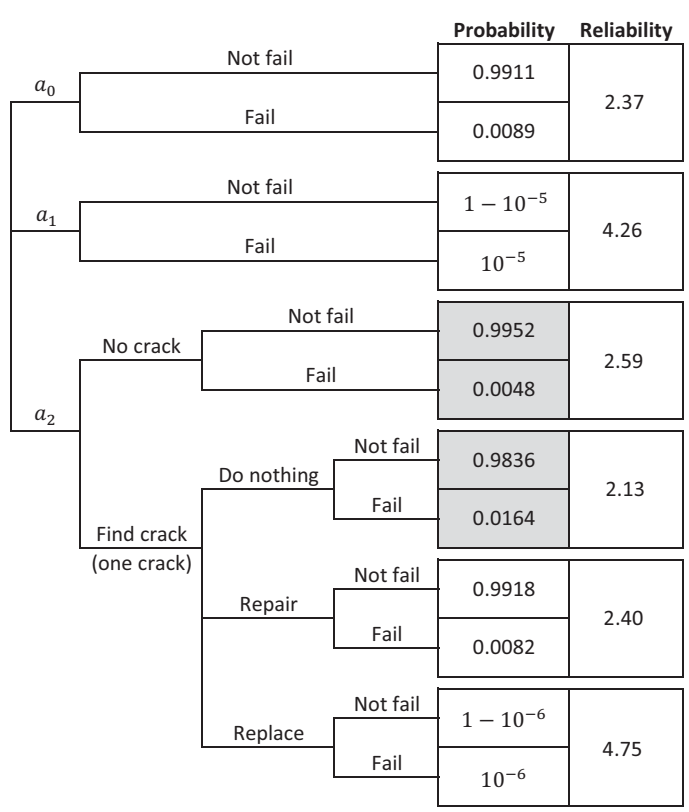

Fig. 8. The updated reliability of the subsystem

\subsection{Consequences}

Here the consequences are eventuated based on cost. Tentative values were assigned in Table 3 for both actions and outcomes. They are not monetary values, instead, they should be seen as ratios between costs. Fatalities are not considered here.

Table 3. Costs for various actions and possible outcomes

\begin{tabular}{l|c|l|c|}
\hline \multicolumn{1}{c|}{ Actions } & Cost & \multicolumn{1}{|c|}{ Outcomes } & Cost \\
\hline$a_{0}:$ do nothing & 0 & Failure & 1000 \\
\hline$a_{1}:$ direct repair & 5 & Repair & $7+n$ \\
\hline$a_{2}:$ inspection & 1 & Replacement & 20 \\
\hline
\end{tabular}

Vote: For Repair, 7 is the basic cost and $n$ refers to the extra cost ncreased with the number of details being fixed. $n$ is within the ange 1 to 8 .

It's obvious that Failure has the highest cost. One reason is that an unexpected failure could happen at any time and will lead to a undesired traffic interruption. The influence to the public can hardly be limited. The potential cost of possible delay, traffic-jam, extra travelling time and accidents is huge. Besides the traffic should be re-routed if possible, which however will leave higher pressure on the alternative routes.

The cost of Repair refers to the cost of doing a corrective maintenance, which fixes the detected defect to restore the fatigue resistance. It's associated with the number of details being fixed.
Replace refers to a higher level of repairing. If multiple serious defects are detected, replacing the subsystem would be more efficient that fixing them one by one. In the following case study, it's assumed that a replacement will be implemented if more than 4 serious defected details are detected.

Both repairing and replacement are assumed to be perfect, which corresponds to a probability of failure $P_{\mathrm{f}}=10^{-6}$.

\subsection{Results}

The risk analysis was summarized as the decision tree in Figure 9 shows. The values of risk is evaluated by multiplying probability of failure with the corresponding consequence, expressed as Eq.(11). In order to make an optimal decision, the value of risk should be ranked and minimized.

$$
\text { Risk }=\text { Probability } \times \text { Consequence }
$$

The second decision node was evaluated first: which action is the optimal if a crack is detected. Under the assumption that only one crack was detected and fixed, the risk of Repair had the lowest value 17.2 and was selected as the optimal choice. However, it's close to the risk level of the scenario 'Find crack $\rightarrow$ Do nothing'. A sensitivity test was performed by changing the number of cracks that have been detected and fixed, and the cost of implementing the repairing. The risk level of 'Do nothing' was marked as the dot line.

When the number of fixed cracks increases, the probability of system failure is monotonically decreasing. Because each fixed crack restores the full fatigue resistance and has individual probability of failure as $P_{\mathrm{f}}=10^{-6}$. However, the risk value shows a different trend. It increases at the beginning because the cost for fixing more cracks are higher; then decreases again, since the probability of failure is getting so small, making the risk contribution from scenario 'Repair $\rightarrow$ Fail' almost negligible.

The basic cost of repairing was adjusted from 6 to 8 . The risk values based on different cost were showing similar trend. However, they can totally influence the final optimal decision. For cost ' $6+n$ ' the risk of repairing is generally lower than risk of 'Do nothing'. For cost ' $7+n$ ', fixing one crack or fixing more than 6 cracks both will make 'Repair' the optimal choice. For cost ' $8+n$ ', only when fixing 8 cracks will have the lowest risk.

After a decision for the second node, the decision tree was updated as shown in Figure 12. The option $a_{1}$ has the lowest risk and is selected as the optimal choice. However, the estimation of consequence could make difference as mentioned before. If the cost of performing a direct repair 


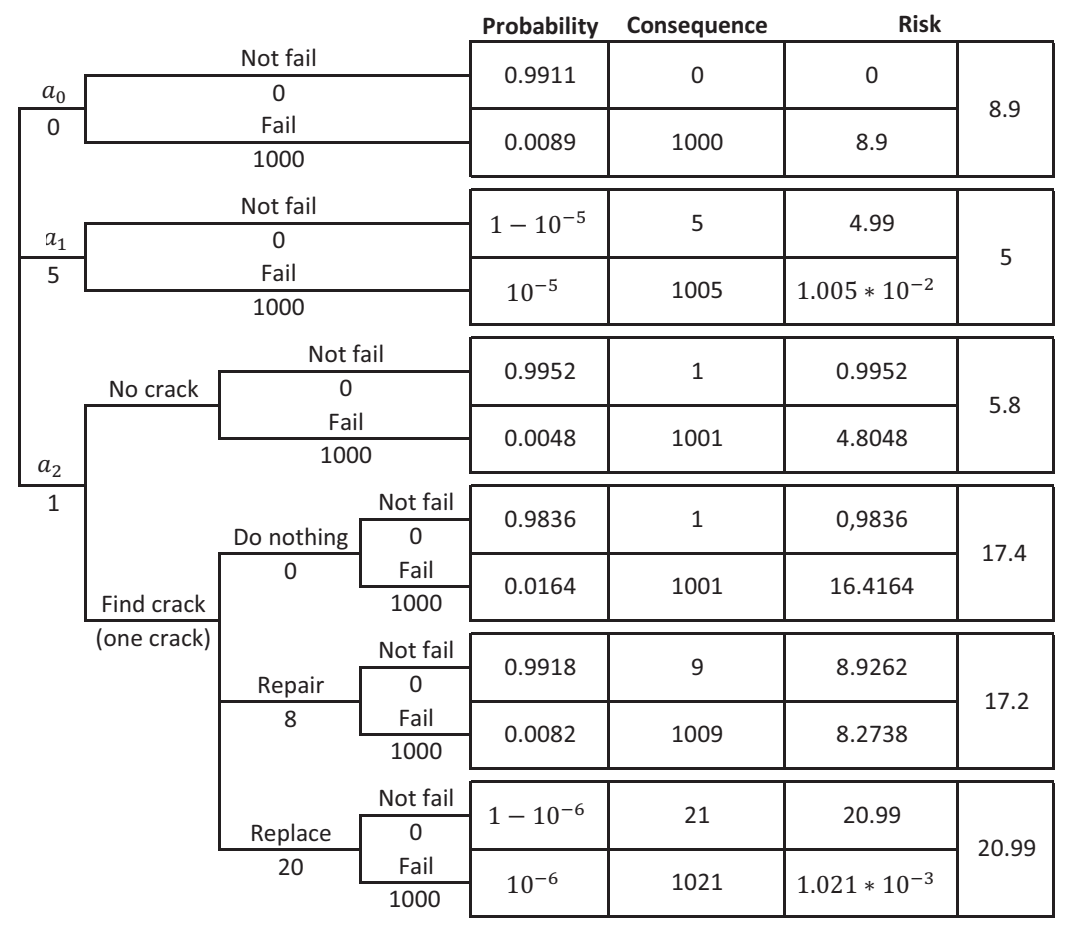

Fig. 9. The result of risk analysis on the selected subsystem. (The calculation for Repair is based on the assumption only one crack is detected and fixed)

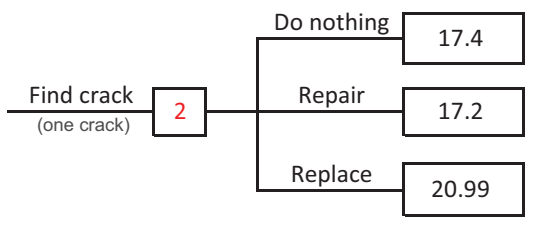

Fig. 10. The second decision node

increases, there is a big chance that it will grow over the risk of $a_{0}$.

If compared to the decision based on the reliability assessment in Section 3.2.2, the optimal decision would be different as Table 4 shows. After finding a crack, the reliability assessment would choose the safest option to replace the whole system even there is only one crack detected. In risk analysis, a more economic option is selected to fix the defection. If there is no crack detected, a direct repair is selected as the optimal intervention by both assessment method, which proves this action economically increases the safety of the subsystem.

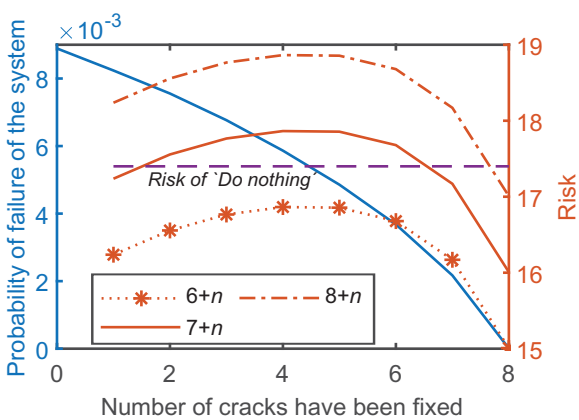

Fig. 11. The risk level changes according to the adjustment of crack number and repair cost. $n$ refers to the number of cracks being detected and fixed.

Table 4. Decisions based on reliability assessment and risk analysis respectively

\begin{tabular}{|c|c|c|}
\hline & No crack & Find crack \\
\hline $\begin{array}{c}\text { Reliability } \\
\text { level }\end{array}$ & $a_{2}$ & Replace \\
\hline Risk level & $a_{2}$ & Repair \\
\hline
\end{tabular}




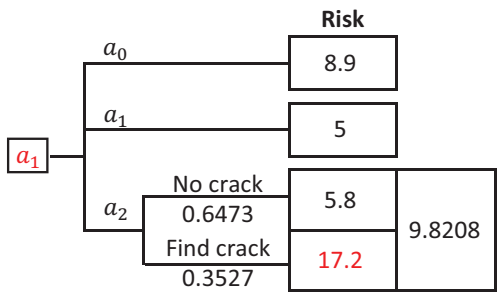

Fig. 12. The first decision node

\section{Conclusions}

A risk analysis was carried out on a subsystem separated from the Rautasjokk Bridge. Multiple possible scenarios were described and evaluated. A decision tree model was established to show the analysis graphically. The following conclusions can be drawn:

- Probability and consequences both influence the evaluation of risk. For bridges it's common to have a small number in the probability of failure. Even if one scenario has extreme severe consequences, the low probability of failure can make the scenario a moderately risky case.

- The decisions based on reliability assessment can be different from that based on risk analysis. The former one only considers the safest option, which could induce unnecessary waste of money and resources, while the latter one evaluates the intervention and consequences together, which could provide a decision that is both economic and safe.

- The values of the consequences matter when it comes to making an optimal decision. A small change of the consequence could directly alter the decision. An accurate estimation of consequences will increase the reliability of the decision.

- The intervention 'Do nothing' is not recommended in the system assessment for bridges. Because up to now there is no clear defined target reliability level for a system, it'll be difficult to define the status of the selected structure.

- Visual inspection brought in new information and provided a noticeable improvement in the system reliability. However, it didn't show much benefit in the value of risk when compared to other alternatives. Because the probability of non-failure and the cost for repairing/replacement are dominating, making the value of risk relatively high.

In this paper only a subsystem has been evaluated and incorporated to demonstrate the decision tree model. A risk-based assessment of the whole bridge is expected and more possible scenarios could be evaluated. In addition, real values of the utilities should be researched and applied in the study, which may influence the risk level at a certain extent.

\section{Acknowledgement}

The financial support of the ongoing project 'Risk-/reliability-based assessment of existing bridges' provided by the KTH Railway Group is gratefully acknowledged.

\section{Citations and References}

\section{References}

BSI (2013). Guide to methods for assessing the acceptability of flaws in metallic structures. $B S$ 7910:2013.

Campbell, L. E., R. J. Connor, J. M. Whitehead, and G. A. Washer (2019). Benchmark for evaluating performance in visual inspection of fatigue cracking in steel bridges. Journal of Bridge Engineering 25(1), 04019128.

Ditlevsen, O. and H. O. Madsen (1996). Structural Reliability Methods, Volume 178. Wiley New York.

Häggström, J. (2015). Bärighetsberäkning: Bro över Rautasjokk KM 1432+883 (in Swedish). Luleå Tekniska Universitet.

Haimes, Y. Y. (2005). Risk modeling, assessment, and management, Volume 40. John Wiley \& Sons.

Hobbacher, A. (1992). Stress intensity factors of plates under tensile load with welded-on flat side gussets. Engineering Fracture Mechanics 41(6), 897-905.

JCSS (2011). Probabilistic Model Code. Joint Committee on Structural Safety (JCSS).

Kwon, K. and D. M. Frangopol (2010). Bridge fatigue reliability assessment using probability density functions of equivalent stress range based on field monitoring data. International Journal of Fatigue 32(8), 1221-1232.

Leander, J. and M. Al-Emrani (2016). Reliabilitybased fatigue assessment of steel bridges using LEFM-A sensitivity analysis. International Journal of Fatigue 93, 82-91.

Leander, J., M. Aygül, and B. Norlin (2013). Refined fatigue assessment of joints with welded in-plane attachments by LEFM. International Journal of Fatigue 56, 25-32.

Newman Jr, J. and I. Raju (1981). An empirical stress-intensity factor equation for the surface crack. Engineering Fracture Mechanics 15(12), 185-192.

Paris, P. C. (1961). A rational analytic theory of fatigue. The Trend in Engineering 13, 9.

Schneider, R., S. Thöns, and D. Straub (2017). Reliability analysis and updating of deteriorating systems with Subset Simulation. Structural Safety 64, 20-36. 